\title{
SOBOLEV ESTIMATES FOR THE LOCAL EXTENSION OF BOUNDARY HOLOMORPHIC FORMS ON REAL HYPERSURFACES IN $\mathbb{C}^{n}$
}

\author{
SANGHYUn ChO
}

\begin{abstract}
Let $M$ be a smooth real hypersurface in complex space of dimension $n, n \geq 3$, and assume that the Levi-form at $z_{0}$ on $M$ has at least $(q+1)$-positive eigenvalues, $1 \leq q \leq n-2$. We estimate solutions of the local $\bar{\partial}$-closed extension problem near $z_{0}$ for $(p, q)$-forms in Sobolev spaces. Using this result, we estimate the local solution of tangential Cauchy-Riemann equation near $z_{0}$ in Sobolev spaces.
\end{abstract}

\section{Introduction}

For a set $D \subset \mathbb{C}^{n}$, we denote the vector space of smooth $(p, q)$-forms on $D$ by $\bigwedge^{p, q}(D)$. Let $\mathcal{M}$ be a smooth real hypersurface in $\mathbb{C}^{n}$ with a smooth defining function $\rho$, and let $\mathcal{B}^{p, q}(\mathcal{M})$ be the restriction of $\bigwedge^{p, q}\left(\mathbb{C}^{n}\right)$ to $\mathcal{M}$ which are pointwise orthogonal to the ideal generated by $\bar{\partial} \rho$. In the sequel, we let $z_{0} \in \mathcal{M}$ be a fixed point and $V$ be a neighborhood of $z_{0}$ in $\mathbb{C}^{n}$ where $\rho$ is defined. For each open set $U \subset V, z_{0} \in U$, we set $U^{-}=\{z \in U ; \rho(z) \leq 0\}$ and $U^{+}=\{z \in U ; \rho(z) \geq 0\}$.

If there exists a neighborhood $U \subset V, z_{0} \in U$, such that for any $\alpha \in$ $\mathcal{B}^{p, q}(\mathcal{M} \cap U)$ with $\bar{\partial}_{b} \alpha=0$ on $\mathcal{M} \cap U$, there exists a smooth $(p, q)$-form $\tilde{\alpha} \in$ $\wedge^{p, q}\left(U^{-}\right)$with $\bar{\partial} \tilde{\alpha}=0$ in $U^{-}$and $(\tilde{\alpha}-\alpha) \wedge \bar{\partial} \rho=0$ on $\mathcal{M} \cap U$, then we say one-sided weak $\bar{\partial}$-closed extension problem is locally solvable.

The $\bar{\partial}$-closed extension problem and the local solvability of the tangential Cauchy-Riemann equation for functions were first introduced in two papers by Hans Lewy $[17,18]$. For the case when $\mathcal{M}$ is the boundary of a smoothly bounded domain $\Omega$ in $\mathbb{C}^{n}$, the global $\bar{\partial}$-closed extension problem for forms from $\mathcal{M}$ to the domain $\Omega$ was studied by J. J. Kohn and H. Rossi [14], who first introduced the $\bar{\partial}_{b}$-complex. They showed that a global $\bar{\partial}$-closed extension exists for any $(p, q)$-form from the boundary $\mathcal{M}=b \Omega$ to the domain $\Omega$ in a complex manifold if $\Omega$ satisfies the condition $Z(n-q-1)$ at all points of $b \Omega$. Analogous

Received January 19, 2012; Revised September 19, 2012.

2010 Mathematics Subject Classification. Primary 32W05; Secondary 32W10.

Key words and phrases. tangential Cauchy-Riemann equation, boundary holomorphic forms. 
result was obtained by Henkin and Leiterer [13] using kernel methods. For the case when $\Omega$ is a bounded pseudoconvex domain in $\mathbb{C}^{n}$, Shaw and Boas $[19,3]$ constructed a two-sided $\bar{\partial}$-closed extension for $\bar{\partial}_{b}$-closed forms near $b \Omega$ using the $L^{2}$-Cauchy problem for $\bar{\partial}$, and solved $\bar{\partial}_{b}$-problem on the boundary.

For the local extension problem, Andreotti and Hill [1] solved the local weak $\bar{\partial}$-closed extension problem when the Levi-form at $z_{0} \in \mathcal{M}$ satisfies the condition $Y(q)$. Under the same assumption, Boggess and Shaw [4] proved the same result using integral kernel method. Recall that we say $\mathcal{M}$ satisfies condition $Y(q)$ at $z_{0}$ if the Levi form of $\mathcal{M}$ at $z_{0}$ has either $\max \{n-q, q+1\}$ eigenvalues of the same sign or $\min \{n-q, q+1\}$ positive and $\min \{n-q, q+1\}$ negative eigenvalues. Thus when $(n-q)>(q+1)$, we need $(q+1)$ mixed (positive and negative) eigenvalues for the condition $Y(q)$ to be satisfied. In [8], Cho and Choi proved the one-sided smooth extension problem (without estimates) when the Levi-form at $z_{0} \in \mathcal{M}$ has at least $(q+1)$ positive eigenvalues.

Note that the estimates of the solutions of these extension problems in various spaces, such as $C^{k}, L^{p}$, Lipschitz or Sobolev spaces, have many applications in the study of complex analysis. For example, function theories on a bounded domain $D \subset \mathbb{C}^{n}$ or the embeddability of abstract $C R$ structures $[5,6,15,21]$.

For a set $W \subset \mathbb{C}^{n}$, we denote the Sobolev norm of order $s$ on $W$ by $\|\cdot\|_{s, W}$. In [10], the author proved the local extension problem, with estimates in Sobolev spaces, for $\bar{\partial}_{b}$-closed $(0,1)$-forms on real hypersurfaces $\mathcal{M}$ in $\mathbb{C}^{n}$ when the Leviform at $z_{0} \in \mathcal{M}$ has two positive eigenvalues. Therefore, it is natural to ask the local extension problem, with estimates in Sobolev spaces, for $(p, q)$-forms when the Levi-form at $z_{0} \in \mathcal{M}$ has at least $(q+1)$ positive eigenvalues (not mixed). In this case, the condition $Y(q)$ is not satisfied when $n-q>q+1$. The following theorem answers this problem.

Theorem 1.1. Let $\mathcal{M}$ be a smooth hypersurface in $\mathbb{C}^{n}, n \geq 3$, with smooth defining function $\rho$ and suppose that the Levi-form at $z_{0} \in \mathcal{M}$ has at least $(q+1)$ positive eigenvalues, $1 \leq q \leq n-2$. Then there is a neighborhood $U_{0}$ of $z_{0}$ such that for any $\alpha \in \mathcal{B}^{p, q}\left(\mathcal{M} \cap U_{0}\right)$, satisfying $\bar{\partial}_{b} \alpha=0$ on $\mathcal{M} \cap U_{0}$, there exists $\tilde{\alpha} \in \bigwedge^{p, q}\left(U_{0}^{-}\right)$such that $\bar{\partial} \tilde{\alpha}=0$ on $U_{0}^{-}$and $(\tilde{\alpha}-\alpha) \wedge \bar{\partial} \rho=0$ on $\mathcal{M} \cap U_{0}$. Also, if $U \subset \subset U_{0}$ is a neighborhood of $z_{0}$ and if we let $\chi \in C_{0}^{\infty}\left(U_{0}\right)$ with $\chi=1$ on $U$, then for each real $s \geq 0$, $\tilde{\alpha}$ satisfies the estimate:

$$
\|\tilde{\alpha}\|_{s+\frac{1}{2}, U^{-}} \leq C_{s}\|\chi \alpha\|_{s+\frac{q+1}{2}, \mathcal{M}} .
$$

We note that the estimate (1.1) is comparable to the case when $q=1$ in [10]. We also note that there are well-known non-solvability results of tangential Cauchy-Riemann equation for $n=2$ [18] and for $q=n-1$ [11]. Note, however, that the local $\bar{\partial}$-closed extension problem and the local solvability of $\bar{\partial}_{b}$ equation are closely related $[19,3]$. Using the results of Theorem 1.1, we solve the local $\bar{\partial}_{b}$-equation in Sobolev spaces.

Theorem 1.2. Let $\mathcal{M}, z_{0} \in \mathcal{M}$ and $U_{0}$ be as in Theorem 1.1. Also, assume that $\mathcal{M}$ is pseudoconvex near $z_{0} \in \mathcal{M}$. Then there is a neighborhood $W$ of 
$z_{0}, W \subset \subset \mathcal{M} \cap U_{0}$, such that for any $\alpha \in \mathcal{B}^{p, q}\left(\mathcal{M} \cap U_{0}\right)$ satisfying $\bar{\partial}_{b} \alpha=0$ on $\mathcal{M} \cap U_{0}$ and for each real $s \geq 0$, there exists $u_{s} \in \mathcal{B}^{(p, q-1)}(W)$ such that $\bar{\partial}_{b} u_{s}=\alpha$ on $W$ and satisfies the estimate:

$$
\left\|u_{s}\right\|_{s, W} \leq C_{s}\|\alpha\|_{s+\frac{q+1}{2}, \mathcal{M} \cap U_{0}^{-}} .
$$

Remark 1.3. In Theorem 1.1, the differentiability assumption $\alpha \in C^{\infty}$ can be weakened to $\alpha \in H^{s+\frac{q+1}{2}}\left(\mathcal{M} \cap U_{0}\right)$ to get $\tilde{\alpha} \in H^{s+\frac{1}{2}}\left(M \cap U_{0}\right)$, and similarly for Theorem 1.2.

Note that the weak extension problem is a Cauchy problem to preserve the boundary values in tangential direction. This means that we have to solve $\bar{\partial}^{*}$ equation instead of $\bar{\partial}$-equation. Let $D$ be a smoothly bounded pseudoconvex domain in $\mathbb{C}^{k}$ and let $\alpha \in \mathcal{B}^{p, q}(b D)$, where $0 \leq p \leq k$ and $1 \leq q \leq k-1$. Note that a necessary and sufficient condition for the extension problem to be solved is:

$$
\int_{b D} \alpha \wedge \psi=0
$$

for every $\psi \in \bigwedge^{k-p, k-q-1}(D) \cap \operatorname{Ker}(\bar{\partial})([7]$, Theorem 9.2.1). We also note that (1.2) is equivalent to the condition $\bar{\partial}_{b} \alpha=0$ for $q \leq k-2$.

\section{Preliminaries}

Let $\Omega$ be a domain in $\mathbb{C}^{k}$ with smooth boundary and let $\bar{\partial}$ be the CauchyRiemann operator on $\Omega$ and let $N_{(p, q)}$ denote the Neumann operator for $(p, q)$ forms. We also let $\mathcal{C}^{p, q}(\Omega)$ be the collection of forms $\phi \in \Lambda^{p, q}(\Omega)$ such that $\phi \wedge \bar{\partial} r=0$ on $b \Omega$, where $r$ is a smooth defining function for $\Omega$. Let $I$ be an open ball in $\mathbb{R}^{d}$ and let $|I|$ denote the diameter of $I$, and let $H_{s, l}(\Omega \times I)$ be the Sobolev space of order $s$ on $\Omega$ and of order $l$ in $I$ with the norm denoted by $\|\cdot\|_{s, l}$. We state a theorem (Theorem 1.7 in [9]) for the smooth dependence of solutions of the $\bar{\partial}$-Neumann problem with respect to a parameter $\tau \in I$.

Theorem 2.1. Let $\left\{\Omega_{\tau}\right\}_{\tau \in I}$ be a smooth family of diffeomorphic strongly pseudoconvex domains in $\mathbb{C}^{k}$ and suppose that $\left\{\alpha_{\tau}\right\}_{\tau \in I}$ is a family of $(p, q)$-forms on $\left\{\Omega_{\tau}\right\}_{\tau \in I}$ such that $\alpha_{\tau} \in R\left(\bar{\partial}_{\tau}\right)$, the range of $\bar{\partial}_{\tau}$, for each $\tau \in I$, where $|I|$ is sufficiently small. Then for each real number $s \geq-1 / 2$ and for each nonnegative integer $l$, there is $C_{s, l}>0$ such that the Neumann solution $U_{\tau}$ of $\square U_{\tau}=\alpha_{\tau}$ and the canonical solution $u_{\tau}=\bar{\partial}^{*} U_{\tau}$ of $\bar{\partial} u_{\tau}=\alpha_{\tau}$ on each $\Omega_{\tau}$, $\tau \in I$, satisfy

$$
\|U\|_{s+1, l},\|u\|_{s+1 / 2, l} \leq C_{s, l} \sum_{r=0}^{l}\|\alpha\|_{s+l-r, r}
$$

where $\alpha \in H_{s+l-r, r}(\Omega \times I), 0 \leq r \leq l$, and where $U:=\left\{u_{\tau}\right\}_{\tau \in I}$ and $\alpha:=$ $\left\{\alpha_{\tau}\right\}_{\tau \in I}$. 
Let $(\mathcal{M}, \rho)$ be as in Section 1 , and assume that the Levi-form at $z_{0} \in \mathcal{M}$ has $k \geq 1$ positive eigenvalues. To prove the solvability of the local extension problem, we need to construct a smooth family of strongly convex domains near $z_{0} \in \mathcal{M}$ which are foliated in the side $\rho \leq 0$ and make up a neighborhood $U_{0}^{-}$of $z_{0} \in \mathcal{M}$. We first prove the following lemma, which describes the local geometry of $\mathcal{M}$ near $z_{0}$ in terms of local coordinates.

Lemma 2.2. Let $\mathcal{M}$ be a smooth hypersurface in $\mathbb{C}^{n}$ and assume that the Leviform at $z_{0} \in \mathcal{M}$ has $k \geq 1$ positive eigenvalues. There is a special coordinate $z=\left(z_{1}, \ldots, z_{n}\right)$ defined in a neighborhood of $z_{0}$ and new defining function $\rho$ of $\mathcal{M}$ which can be written, in new coordinates, by

$$
\rho(z)=\left|z_{n}\right|^{2}-1+\sum_{i=1}^{k}\left|z_{i}\right|^{2}+\sum_{i=k+1}^{n-1} \lambda_{i}\left|z_{i}\right|^{2}+\mathcal{O}\left(\left|z-z_{0}\right|^{3}\right) .
$$

Proof. Let $\rho$ be a smooth defining function of $\mathcal{M}$. By a standard method of holomorphic coordinate changes, we have special coordinates $u=\left(u^{\prime}, u^{\prime \prime}\right)$, $u^{\prime}=\left(u_{1}, \ldots, u_{k}\right), u^{\prime \prime}=\left(u_{k+1}, \ldots, u_{n}\right), u\left(z_{0}\right)=0$ and the Taylor expansion near $z_{0}=0$ can be written as:

$\rho(u)=u_{n}+\bar{u}_{n}+\sum_{i=1}^{k}\left|u_{i}\right|^{2}+\sum_{i=k+1}^{n-1} \lambda_{i}\left|u_{i}\right|^{2}+\sum_{k=1}^{n} c_{k} u_{k} \bar{u}_{n}+\sum_{k=1}^{n} \bar{c}_{k} \bar{u}_{k} u_{n}+\mathcal{O}\left(|u|^{3}\right)$,

where each $\lambda_{i}$ is a real number and $\mathcal{O}\left(|u|^{3}\right)$ is the remainder whose first and second derivatives vanishes at 0 . Set $r(u)=\rho(u) \cdot\left(1-\sum_{k=1}^{n} c_{k} u_{k}-\sum_{k=1}^{n} \bar{c}_{k} \bar{u}_{k}\right)$, where $\left(1-2 \operatorname{Re} \sum_{k=1}^{n} c_{k} u_{k}\right)>1 / 2$ in a neighborhood of the origin. Therefore, $r$ is a new local defining function of $\mathcal{M}$ near $z_{0} \in \mathcal{M}$ and can be written as

$r(u)=\left(u_{n}-\sum_{k=1}^{n} c_{k} u_{k} u_{n}\right)+\left(\bar{u}_{n}-\sum_{k=1}^{n} \bar{c}_{k} \bar{u}_{k} \bar{u}_{n}\right)+\sum_{i=1}^{k}\left|u_{i}\right|^{2}+\sum_{i=k+1}^{n-1} \lambda_{i}\left|u_{i}\right|^{2}+\mathcal{O}\left(|u|^{3}\right)$.

Set $w_{j}=u_{j}$ for $j<n$, and $w_{n}=u_{n}-\sum_{k=1}^{n} c_{k} u_{k} u_{n}$. In $w$ coordinates, $r(w)$ has the representation:

$$
r(w)=w_{n}+\bar{w}_{n}+\sum_{i=1}^{k}\left|w_{i}\right|^{2}+\sum_{i=k+1}^{n-1} \lambda_{i}\left|w_{i}\right|^{2}+\mathcal{O}\left(|w|^{3}\right) .
$$

Set $\tilde{r}(w)=r(w) \cdot\left(1+\left(w_{n}+\bar{w}_{n}\right) / 2\right)$, where $\left.1+\left(w_{n}+\bar{w}_{n}\right) / 2\right)>1 / 2$ near $z_{0}=0$. Then $\tilde{r}(w)$ can be written as:

$$
\tilde{r}(w)=w_{n}+\bar{w}_{n}+\frac{1}{2}\left(w_{n}^{2}+\bar{w}_{n}^{2}\right)+\left|w_{n}\right|^{2}+\sum_{i=1}^{k}\left|w_{i}\right|^{2}+\sum_{i=k+1}^{n-1} \lambda_{i}\left|w_{i}\right|^{2}+\mathcal{O}\left(|w|^{3}\right) .
$$

By setting

$$
\tilde{u}_{n}=w_{n}+\frac{1}{2} w_{n}^{2} \text { and } \tilde{u}_{j}=w_{j}, j<n
$$


$\tilde{r}$ can be written, in $\tilde{u}$-coordinates, by:

$$
\tilde{r}(\tilde{u})=\tilde{u}_{n}+\overline{\tilde{u}}_{n}+\left|\tilde{u}_{n}\right|^{2}+\sum_{i=1}^{k}\left|\tilde{u}_{i}\right|^{2}+\sum_{i=k+1}^{n-1} \lambda_{i}\left|\tilde{u}_{i}\right|^{2}+\mathcal{O}\left(|\tilde{u}|^{3}\right) .
$$

Finally, we set $z_{n}=\tilde{u}_{n}+1$ and $z_{j}=\tilde{u}_{j}$ for $j<n$, and denote $\tilde{r}$ by $\rho$. Then $z\left(z_{0}\right)=(0, \ldots, 0,1)$ and in $z$-coordinates, the local defining function $\rho$ can be written as:

$$
\rho(z)=\left|z_{n}\right|^{2}-1+\sum_{i=1}^{k}\left|z_{i}\right|^{2}+\sum_{i=k+1}^{n-1} \lambda_{i}\left|z_{i}\right|^{2}+\mathcal{O}\left(\left|z-z_{0}\right|^{3}\right)
$$

near $z_{0}=z\left(z_{0}\right)=(0, \ldots, 0,1)$.

Set $z=\left(z^{\prime}, z^{\prime \prime}\right)$, where $z^{\prime}=\left(z_{1}, \ldots, z_{k}\right)$ and $z^{\prime \prime}=\left(z_{k+1}, \ldots, z_{n}\right)$. In the following proposition, we regard $z^{\prime \prime} \in \mathbb{C}^{n-k}$ as a parameter variable near $t_{0}^{\prime \prime}:=$ $z_{0}^{\prime \prime}=(0, \ldots, 0,1) \in \mathbb{C}^{n-k}$ and construct a family of strongly convex domains. In Section 3, we will apply Theorem 2.1 to this parameter family of domains.

Proposition 2.3. Let $(\mathcal{M}, \rho)$ and $z_{0}$ be as in Lemma 2.2. Then there exist a small open ball $I:=B_{\sigma_{0}}\left(t_{0}^{\prime \prime}\right) \subset \mathbb{C}^{n-k}$ for a small $\sigma_{0}>0$ and a family of bounded strongly convex domains $\left\{\Omega_{t^{\prime \prime}}\right\}_{t^{\prime \prime} \in I}$ in $\mathbb{C}^{k}$, and $\Omega_{t^{\prime \prime}}$ is diffeomorphic to $\Omega_{t_{0}^{\prime \prime}}$ for each $t^{\prime \prime} \in I$ with diameters being strictly bounded from below (say, by $\left.\sigma_{0}^{17 / 48}\right)$, and foliate into the part $\rho \leq 0$ making up a neighborhood $U_{0}^{-}$of $z_{0}$.

Proof. For a sufficiently small $\sigma>0$ to be determined, let $B_{\sigma}\left(t_{0}^{\prime \prime}\right) \subset \mathbb{C}^{n-k}$ be a ball of radius $\sigma>0$ centered at $t_{0}^{\prime \prime}$. For any fixed $t^{\prime \prime}=\left(t_{k+1}, \ldots, t_{n}\right) \in B_{\sigma}\left(t_{0}^{\prime \prime}\right)$ and for each $\left|z^{\prime}\right|<\sigma^{1 / 4}$, set $\tilde{\rho}\left(z^{\prime}, t^{\prime \prime}\right)=\rho\left(z^{\prime}, t_{k+1}, \ldots, t_{n-1}, t_{n}-\sigma^{1 / 3} z_{1}\right)$. In view of $(2.2)$, we can write

$$
\begin{aligned}
\tilde{\rho}\left(z^{\prime}, t^{\prime \prime}\right)= & \left(\left|t_{n}\right|^{2}-1\right)-2 \sigma^{1 / 3} \operatorname{Re}\left(z_{1} \bar{t}_{n}\right)+\sigma^{2 / 3}\left|z_{1}\right|^{2}+\sum_{i=1}^{k}\left|z_{i}\right|^{2} \\
& +\sum_{i=k+1}^{n-1} \lambda_{i}\left|t_{i}\right|^{2}+\mathcal{O}\left(\left|z-z_{0}\right|^{3}\right) .
\end{aligned}
$$

For $t^{\prime \prime} \in B_{\sigma}\left(t_{0}^{\prime \prime}\right)$, we set

$$
r_{t^{\prime \prime}}^{\sigma}\left(z^{\prime}\right):=\left(1-\left|t_{n}\right|^{2}\right)+2 \sigma^{1 / 3} \operatorname{Re}\left(z_{1} \bar{t}_{n}\right)-\sum_{i=k+1}^{n-1} \lambda_{i}\left|t_{i}\right|^{2}+\mathcal{O}\left(\left|z-z_{0}\right|^{3}\right) .
$$

Then when $t^{\prime \prime}=t_{0}^{\prime \prime}=(0, \ldots, 0,1)$, i.e., at the center of the ball $B_{\sigma}\left(t_{0}^{\prime \prime}\right)$, we have

$$
r_{t_{0}^{\prime \prime}}^{\sigma}\left(z^{\prime}\right)=2 \sigma^{1 / 3} \operatorname{Re}\left(z_{1}\right)+\mathcal{O}\left(\left|z^{\prime}\right|^{3}\right)>0
$$


for an appropriate $z_{1}$ (say, at $\operatorname{Re} z_{1}=\sigma^{3 / 8}$ ) provided $\sigma>0$ is sufficiently small. Therefore, it follows that

$$
\Omega_{t_{0}^{\prime \prime}}:=\left\{z^{\prime} \in B_{\sigma^{1 / 4}}\left(z_{0}^{\prime}\right) ; \sigma^{2 / 3}\left|z_{1}\right|^{2}+\sum_{i=1}^{k}\left|z_{i}\right|^{2}<r_{t_{0}^{\prime \prime}}^{\sigma}\left(z^{\prime}\right)\right\}
$$

is a non-empty strongly convex domain contained in the side of $\rho \leq 0$. Note that $\Omega_{t_{0}^{\prime \prime}}$ is a small deformation of a ball whose radius is bigger than or equal to $\sigma^{17 / 48}$. Also we see that $z_{0} \in b \Omega_{t_{0}^{\prime \prime}} \subset \mathcal{M}$ and $\Omega_{t_{0}^{\prime \prime}}$ is the central slice of the side $\rho \leq 0$.

For any $t^{\prime \prime}=\left(t_{k+1}, \ldots, t_{n}\right) \in B_{\sigma}\left(t_{0}^{\prime \prime}\right)$, we note that $\left|t_{n}-1\right|<\sigma$. Hence $r_{t^{\prime \prime}}^{\sigma}$ is a small (of size less than $\sigma$ ) perturbation of $r_{t_{0}^{\prime \prime}}^{\sigma}$. Therefore, as for the $r_{t_{0}^{\prime \prime}}^{\sigma}$ case, it follows that $r_{t^{\prime \prime}}^{\sigma}\left(z^{\prime}\right)>0$ for some $z^{\prime}$ and hence for each $t^{\prime \prime} \in B_{\sigma}\left(t_{0}^{\prime \prime}\right)$,

$$
\Omega_{t^{\prime \prime}}:=\left\{z^{\prime} \in \mathbb{C}^{k} ; \sigma^{2 / 3}\left|z_{1}\right|^{2}+\sum_{i=1}^{k}\left|z_{i}\right|^{2}<r_{t^{\prime \prime}}^{\sigma}\left(z^{\prime}\right)\right\}
$$

is a nonempty strongly convex domain in $\mathbb{C}^{k}$ contained in the side of $\{z ; \rho(z)<$ $0\}$ and $b \Omega_{t^{\prime \prime}} \subset \mathcal{M}$, and the diameter of $\Omega_{t^{\prime \prime}}$ is bigger than or equal to $\sigma^{17 / 48}$ provided $\sigma$ is sufficiently small. Let us fix $\sigma=\sigma_{0}$ satisfying the above conditions, and set $I:=B_{\sigma_{0}}\left(t_{0}^{\prime \prime}\right) \subset \mathbb{C}^{n-k}$ and

$$
U_{0}^{-}:=\bigcup_{t^{\prime \prime} \in I} \bar{\Omega}_{t^{\prime \prime}} \times\left\{t^{\prime \prime}\right\}
$$

This proves the proposition.

Remark 2.4. Note that $\operatorname{Re} z_{1} \lesssim \sigma^{1 / 3}$ if $z^{\prime} \in \Omega_{t^{\prime \prime}}$, which forces that $\left|z^{\prime}\right| \lesssim \sigma^{1 / 3}$, that is, $\Omega_{t^{\prime \prime}} \subset B_{\sigma^{7 / 24}}\left(z_{0}\right) \subset B_{\sigma^{1 / 4}}\left(z_{0}\right)$ provided $\sigma$ is sufficiently small. Also, in view of our construction, we may take $\sigma_{0}$ sufficiently small so that Theorem 2.1 holds for $I=B_{\sigma_{0}}$ and $U_{0}^{-} \subset B_{\sigma_{0}^{1 / 4}}\left(z_{0}\right)$.

Remark 2.5. With the special coordinates $z=\left(z^{\prime}, t^{\prime \prime}\right)$ defined in $(2.3)$, set $\tilde{t}_{n}=1+\frac{1}{2} \sigma^{7 / 24}$, and for each $\left|t_{j}\right|<\sigma^{1 / 3}, j=k+1, \ldots, n-1$, set $\tilde{t}^{\prime \prime}=$ $\left(t_{k+1}, \ldots, t_{n-1}, \tilde{t}_{n}\right)$. For each $\left|z^{\prime}\right|<\sigma^{1 / 3}$, we then have $r_{\tilde{t}^{\prime \prime}}^{\sigma}\left(z^{\prime}\right) \approx-\sigma^{7 / 24}$, and hence $\tilde{\rho}\left(z^{\prime}, \tilde{t}^{\prime \prime}\right)>0$. Set $\tilde{D}_{\sigma}^{\prime \prime}:=\left\{t^{\prime \prime} \in \mathbb{C}^{n-k} ;\left|t_{j}\right|<\sigma^{1 / 3}, k+1 \leq j \leq\right.$ $\left.n-1,\left|t_{n}-\tilde{t}_{n}\right|<\sigma\right\}$. Then for each $t^{\prime \prime} \in \tilde{D}_{\sigma}^{\prime \prime}$ and $\left|z^{\prime}\right|<\sigma^{1 / 3}$, it follows that $\tilde{\rho}_{t^{\prime \prime}}\left(z^{\prime}\right)>0$, and hence $\Omega_{t^{\prime \prime}}$ is an empty set when $t^{\prime \prime} \in \tilde{D}_{\sigma}^{\prime \prime}$.

\section{3. $\bar{\partial}$-closed extension for $(p, q)$-forms}

To prove the local extension theorem, we use the local decomposition of the set $U_{0}^{-}$considered in (2.4) and use Proposition 2.3 with $k=q+1$. We will solve the $\vartheta$-equation to correct the terms and use the estimates (2.1) on parameter 
variables $z^{\prime \prime}=t^{\prime \prime} \in I$, where $I$ is defined as in (2.4). Set $\mathcal{K}=\{1, \ldots, q+1\}$ and $\mathcal{K}^{c}=\{q+2, \ldots, n\}$. For a smooth function $f$ defined in $\mathbb{C}^{n}$, we define

$$
\bar{\partial}_{\mathcal{K}} f=\sum_{j=1}^{q+1} \frac{\partial f}{\partial \bar{z}_{j}} d \bar{z}_{j} \quad \text { and } \quad \bar{\partial}_{\mathcal{K}^{c}} f=\sum_{j=q+2}^{n} \frac{\partial f}{\partial \bar{z}_{j}} d \bar{z}_{j} .
$$

We can extend this definition for arbitrary smooth forms.

Since $p$ does not play any important role in the estimates, we set $p=0$, i.e., we consider only the cases of $\bigwedge^{0, q}(W)$, where $W$ is an appropriate set. We recall that $\|\cdot\|_{s, k, W}$ is the Sobolev space of order $s$ in $z^{\prime} \in \mathbb{C}^{q+1}$ variables and of order $k$ in $z^{\prime \prime} \in I \subset \mathbb{C}^{n-q-1}$ variables. We also note that $\bigwedge^{p, q}\left(\Omega_{z^{\prime \prime}}\right)$ and $\Lambda^{p, q}\left(b \Omega_{z^{\prime \prime}}\right)$ are defined on $\Omega_{z^{\prime \prime}} \subset \mathbb{C}^{q+1}$, and that every summation will be over strictly increasing indices. In the sequel, the constants, such as $C_{s}$ or $C_{s, k}$, depend only on $s$ or $k$ and can vary line-to-line while we estimate.

Proposition 3.1. Let $\mathcal{M}$ be a smooth real hypersurface in $\mathbb{C}^{n}, n \geq 3$, with smooth defining function $\rho$ defined in a neighborhood $V$ of $z_{0} \in \mathcal{M}$, and suppose that the Levi-form at $z_{0}$ has at least $(q+1)$ positive eigenvalues, $1 \leq q \leq n-2$. Then there is a neighborhood $U_{0}, z_{0} \in U_{0}$, such that for any $\alpha \in \mathcal{B}^{0, q}\left(\mathcal{M} \cap U_{0}\right)$, there are $\tilde{\alpha}_{j} \in \Lambda^{0, q}\left(U_{0}^{-}\right), 0 \leq j \leq q+1$, such that $\left(\tilde{\alpha}_{j}-\alpha\right) \wedge \bar{\partial} \rho=0$ on $\mathcal{M} \cap U_{0}$ and $\bar{\partial} \tilde{\alpha}_{j}$ can be written as

$$
\bar{\partial} \tilde{\alpha}_{j}=\sum_{\substack{I \subset \mathcal{K}, J \subset \mathcal{K}^{c} \\|I|+|J|=q+1,|J| \geq j}} \alpha_{I J}^{j} d \bar{z}^{I} \wedge d \bar{z}^{J}
$$

on $U_{0}^{-}$for some smooth functions $\alpha_{I J}^{j}$. Also, if $U \subset \subset U_{0}$ is a neighborhood of $z_{0}$ and if we let $\chi \in C_{0}^{\infty}\left(U_{0}\right)$ with $\chi=1$ on $U$, then for each real $s \geq 0, \tilde{\alpha}_{j}$ satisfy the estimate

$$
\left\|\tilde{\alpha}_{j}\right\|_{s+\frac{1}{2}, U^{-}} \leq C_{s}\|\chi \alpha\|_{s+\frac{j}{2}, \mathcal{M}}
$$

for each $0 \leq j \leq q+1$.

Proof. Let us take $U_{0} \subset B_{\sigma_{0}^{1 / 4}} \subset V$ as defined in (2.4) where special frames are defined on $V$. By shrinking $I$ if necessary, we may assume that Theorem 2.1 holds on $U_{0}$. Using Theorem 2.1, we shall construct $\tilde{\alpha}_{j}$ inductively satisfying (3.1) and (3.2). From Lemma 9.3.3 in [7], there is $\tilde{\alpha}_{0}:=E \alpha \in \Lambda^{0, q}\left(U_{0}\right)$, $E \alpha=\alpha$ and $\bar{\partial} E \alpha=\mathcal{O}\left(\rho^{\infty}\right)$ on $\mathcal{M} \cap U_{0}$ such that for each real $s$, we have

$$
\left\|\tilde{\alpha}_{0}\right\|_{s, U_{0}^{-}} \leq C_{s}\|\chi \alpha\|_{s-1 / 2, \mathcal{M}} .
$$

Thus (3.1) and (3.2) hold for $j=0$.

Let $U \subset \subset U_{0}$ be a neighborhood of $z_{0}$ and choose a smooth cut-off function $\chi \in C_{0}^{\infty}\left(U_{0}\right)$ with $\chi=1$ on $U$. Replacing $\tilde{\alpha}_{0}$ by $\chi \tilde{\alpha}_{0}$, we may assume that 
$\tilde{\alpha}_{0} \in C_{0}^{\infty}\left(U_{0}\right)$. Let us write

$$
\begin{aligned}
\bar{\partial} \tilde{\alpha}_{0} & =\alpha_{\mathcal{K}}^{0} d \bar{z}^{\mathcal{K}}+\sum_{j=q+2}^{n} \sum_{\substack{|I|=q \\
I \subset \mathcal{K}}} \beta_{I l}^{1} d \bar{z}^{I} \wedge d \bar{z}^{j}+\sum_{\substack{|I|+|J|=q+1,|J| \geq 2 \\
I \subset \mathcal{K}, J \subset \mathcal{K}^{c}}} E_{I J}^{2} d \bar{z}^{I} \wedge d \bar{z}^{J} \\
& :=\alpha^{0}+\beta^{1}+E^{2}
\end{aligned}
$$

and set

$$
g^{0}=\alpha^{0} \text { on } U_{0}^{-} \text {, and } g^{0}=0 \text { on } U_{0}^{+} .
$$

Since $\bar{\partial} \tilde{\alpha}_{0}=\mathcal{O}\left(\rho^{\infty}\right)$ on $\mathcal{M} \cap U_{0}$, it follows that $g^{0} \in C^{\infty}\left(U_{0}\right)$. Similarly, if we define $\beta_{I l}^{1}=E_{I J}^{2}=0$ on $U_{0}^{+}$, it follows that $\beta_{I l}^{1}, E_{I J}^{2} \in C^{\infty}\left(U_{0}\right)$. Note that $g^{0}$ comes from the components of $\bar{\partial}_{\mathcal{K}} \tilde{\alpha}_{0}$. By (3.3), for each real $s \geq-1$ and nonnegative integer $k$, there are $\tilde{C}_{s, k}$ and $C_{s, k}$ such that

$$
\left\|g^{0}\right\|_{s, k, U^{-}} \leq \tilde{C}_{s, k}\left\|\tilde{\alpha}_{0}\right\|_{s+1, k, U^{-}} \leq C_{s, k}\|\chi \alpha\|_{s+k+1 / 2, \mathcal{M}}
$$

To remove $\alpha^{0}$ term in $\bar{\partial} \tilde{\alpha}_{0}$, we try to solve $\bar{\partial}_{\mathcal{K}} u_{0}\left(\cdot, z^{\prime \prime}\right)=g^{0}\left(\cdot, z^{\prime \prime}\right)$ in $\Omega_{z^{\prime \prime}}$ and set $\tilde{\alpha}_{1}\left(\cdot, z^{\prime \prime}\right)=\tilde{\alpha}_{0}\left(\cdot, z^{\prime \prime}\right)-u_{0}\left(\cdot, z^{\prime \prime}\right)$ for each $z^{\prime \prime} \in I$. However, to preserve the boundary condition, it is required that $u_{0}\left(\cdot, z^{\prime \prime}\right) \in \mathcal{C}^{0, q}\left(\Omega_{z^{\prime \prime}}\right)$ for each $z^{\prime \prime} \in I$. This means that we have to solve $\bar{\partial}_{\mathcal{K}^{-}}^{*}$ equation rather than $\bar{\partial}_{\mathcal{K}}$-equation. Since $g^{0}\left(\cdot, z^{\prime \prime}\right)$ is a $(0, q+1)$-form in $\Omega_{z^{\prime \prime}} \subset \mathbb{C}^{q+1}$, it becomes a top degree problem in $\mathbb{C}^{q+1}$ and hence it is required to satisfy (1.2), that is,

$$
F_{h}\left(z^{\prime \prime}\right):=\int_{\Omega_{z^{\prime \prime}}} g^{0}\left(\cdot, z^{\prime \prime}\right) \wedge h(\cdot)=0, \text { for every } h \in C_{(q+1,0)}^{\infty}\left(\mathbb{C}^{q+1}\right) \cap \operatorname{ker}\left(\bar{\partial}_{\mathcal{K}}\right) .
$$

To prove (3.5), we consider the coefficients of the terms of $d \bar{z}^{\mathcal{K}} \wedge d \bar{z}_{l}$ in $\bar{\partial}^{2} \tilde{\alpha}_{0}=0$, where $l \in \mathcal{K}^{c}$. Note that these terms are coming from $\bar{\partial}_{\mathcal{K}^{c}} \alpha^{0}+\bar{\partial}_{\mathcal{K}} \beta^{1}$. Thus we obtain that

$$
\frac{\partial \alpha_{\mathcal{K}}^{0}}{\partial \bar{z}_{l}}=\sum_{j=1}^{q+1} \sum_{\substack{|I|=q \\ I \subset \mathcal{K}}}(-1)^{q+j-1} \frac{\partial \beta_{I l}^{1}}{\partial \bar{z}_{j}}, \quad l=q+2, \ldots, n .
$$

In view of (3.5) and (3.6), it follows, for $l \in \mathcal{K}^{c}$, that

$$
\begin{aligned}
\frac{\partial F_{h}}{\partial \bar{z}_{l}}\left(z^{\prime \prime}\right) & =\frac{\partial}{\partial \bar{z}_{l}} \int_{\mathbb{C}^{q+1}} g^{0}\left(\cdot, z^{\prime \prime}\right) \wedge h \\
& =\int_{\Omega_{z^{\prime \prime}}} \frac{\partial}{\partial \bar{z}_{l}} \alpha_{\mathcal{K}}^{0}\left(\cdot, z^{\prime \prime}\right) \wedge h \\
& =\int_{\Omega_{z^{\prime \prime}}} \sum_{j=1}^{q+1} \sum_{\substack{|I|=q \\
I \subset \mathcal{K}}}(-1)^{q+j-1} \frac{\partial \beta_{I l}^{1}}{\partial \bar{z}_{j}} \wedge h \\
& =\int_{\Omega_{z^{\prime \prime}}} \sum_{j=1}^{q+1} \sum_{\substack{|I|=q \\
I \subset \mathcal{K}}}(-1)^{q+j} \beta_{I l}^{1} \wedge \frac{\partial h}{\partial \bar{z}_{j}}=0
\end{aligned}
$$


because $h(\cdot)$ is holomorphic in $\Omega_{z^{\prime \prime}}$. Here, the first and the second equalities hold because $g^{0}$ is supported in $\Omega_{z^{\prime \prime}} \subset \mathbb{C}^{q+1}$, and the fourth equality holds because $\beta_{I l}^{1}=0$ on $b \Omega_{z^{\prime \prime}}$ (and hence we can perform integration by parts). Therefore, $F_{h}\left(z^{\prime \prime}\right)$ is holomorphic in $\mathbb{C}^{n-q-1}$. Moreover, in view of Remark 2.5, it follows that $F_{h}\left(z^{\prime \prime}\right)=0$ for $z^{\prime \prime} \in \tilde{D}_{\sigma}^{\prime \prime}$ (since $\Omega_{z^{\prime \prime}}$ becomes the empty set), provided $\sigma$ is sufficiently small. Here, $\tilde{D}_{\sigma}^{\prime \prime}$ is the tube defined in Remark 2.5. Thus we see that (3.5) holds.

Set $u_{0}\left(\cdot, z^{\prime \prime}\right)=-*_{\mathcal{K}} \bar{\partial}_{\mathcal{K}} N_{(q+1,0)}^{\mathcal{K}} * \mathcal{K} g^{0}\left(\cdot, z^{\prime \prime}\right), z^{\prime \prime} \in I$, where $N_{(r, s)}^{\mathcal{K}}$ is the Neumann operator for $(r, s)$-forms and $*_{\mathcal{K}}$ is the Hodge star operator on $\Omega_{z^{\prime \prime}}$ for each $z^{\prime \prime} \in I$. Then we have $\bar{\partial}_{\mathcal{K}} u_{0}\left(\cdot, z^{\prime \prime}\right)=g^{0}\left(\cdot, z^{\prime \prime}\right)$ and $u_{0}\left(\cdot, z^{\prime \prime}\right) \in \mathcal{C}^{0, q}\left(\Omega_{z^{\prime \prime}}\right)$ for each $z^{\prime \prime} \in I$. We also note that $u_{0}\left(\cdot, z^{\prime \prime}\right)$ depends smoothly on $z^{\prime \prime} \in I$ and satisfies the estimate (2.1) including the parameter variable $z^{\prime \prime} \in I$. Thus for each real $s \geq-1 / 2$ and nonnegative integer $k$, it follows from (2.1) and (3.4) that

$$
\left\|u_{0}\right\|_{s+\frac{1}{2}, k, U^{-}} \lesssim \sum_{r=0}^{k}\left\|g^{0}\right\|_{s+k-r, r, U^{-}} \lesssim\left\|\tilde{\alpha}_{0}\right\|_{s+1+k, U^{-}} \lesssim\|\chi \alpha\|_{s+\frac{1}{2}+k, \mathcal{M}}
$$

Note that $u_{0} \wedge \bar{\partial}_{\mathcal{K}} \rho=0$ on $\mathcal{M} \cap U_{0}$ because $u_{0}\left(\cdot, z^{\prime \prime}\right) \in \mathcal{C}^{0, q}\left(\Omega_{z^{\prime \prime}}\right)$ for each $z^{\prime \prime} \in I$. We have to correct $u_{0}$ so that the corrected one, $\tilde{u}_{0}$, belongs to $\mathcal{C}^{0, q}(\Omega)$, that is, $\tilde{u}_{0} \wedge \bar{\partial} \rho=0$ on $\mathcal{M} \cap U_{0}$. Since $u_{0} \wedge \bar{\partial}_{\mathcal{K}} \rho=0$ on $\mathcal{M} \cap U_{0}$, we can write

$$
u_{0}\left(\cdot, z^{\prime \prime}\right)=\delta^{0}\left(\cdot, z^{\prime \prime}\right) \wedge \bar{\partial}_{\mathcal{K}} \rho\left(\cdot, z^{\prime \prime}\right)+\rho\left(\cdot, z^{\prime \prime}\right) \gamma^{0}\left(\cdot, z^{\prime \prime}\right)
$$

for some $\delta^{0}\left(\cdot, z^{\prime \prime}\right) \in \mathcal{C}^{0, q}\left(\Omega_{z^{\prime \prime}}\right)$ and $\gamma^{0}\left(\cdot, z^{\prime \prime}\right) \in \mathcal{C}^{0, q+1}\left(\Omega_{z^{\prime \prime}}\right), z^{\prime \prime} \in I$. We may assume that $\delta^{0} \wedge \bar{\partial}_{\mathcal{K}} \rho$ and $\rho \gamma^{0}$ are disjoint, and hence it follows from the estimate in (3.7) that

$$
\left\|\delta^{0}\right\|_{s+\frac{1}{2}, k, U^{-}} \lesssim\left\|u_{0}\right\|_{s+\frac{1}{2}, k, U^{-}} \lesssim\|\chi \alpha\|_{s+\frac{1}{2}+k, \mathcal{M}}
$$

for each real $s \geq-\frac{1}{2}$ and nonnegative integer $k$.

Set $\tilde{u}_{0}=u_{0}+\delta^{0} \wedge \bar{\partial}_{\mathcal{K}^{c}} \rho$. Then one obtains that

$$
\tilde{u}_{0} \wedge \bar{\partial} \rho=\left(\delta^{0} \wedge \bar{\partial}_{\mathcal{K}} \rho+\rho \gamma^{0}+\delta^{0} \wedge \bar{\partial}_{\mathcal{K}^{c}} \rho\right) \wedge \bar{\partial} \rho=0,
$$

on $\mathcal{M} \cap U_{0}$, and hence $\tilde{u}_{0} \in \mathcal{C}^{0, q}\left(U_{0}^{-}\right)$. Now we set $\tilde{\alpha}_{1}=\tilde{\alpha}_{0}-\tilde{u}_{0}$. Then it follows that $\left(\tilde{\alpha}_{1}-\alpha\right) \wedge \partial \rho=-\tilde{u}_{0} \wedge \partial \rho=0$ on $U \cap \mathcal{M}$, and we can write

$$
\bar{\partial} \tilde{\alpha}_{1}=\sum_{l=q+2}^{n} \sum_{\substack{|I|=q \\ I \subset \mathcal{K}}} \tilde{\beta}_{I l}^{1} d \bar{z}^{I} \wedge d \bar{z}^{l}+\sum_{\substack{|I|+|J|=q+1,|J| \geq 2 \\ I \subset \mathcal{K}, J \subset \mathcal{K}^{c}}} \tilde{\beta}_{I J}^{2} d \bar{z}^{I} \wedge d \bar{z}^{J}
$$

for some smooth functions $\tilde{\beta}_{I l}^{1}$ and $\tilde{\beta}_{I J}^{2}$. In view of $(3.3),(3.7)$ and (3.8), one obtains that

$$
\left\|\tilde{\alpha}_{1}\right\|_{s+\frac{1}{2}, k, U^{-}} \lesssim\|\chi \alpha\|_{s+\frac{1}{2}+k, \mathcal{M}}
$$

for each real $s \geq-\frac{1}{2}$ and nonnegative integer $k$. 
By induction, assume that there are $\tilde{\alpha}_{j} \in \bigwedge^{0, q}\left(U_{0}^{-}\right), j \geq 0$, satisfying (3.1) and (3.2), and that for each real $s \geq 0$, the estimate

$$
\left\|\tilde{\alpha}_{j}\right\|_{s+\frac{1}{2}, U^{-}} \leq C_{s}\|\chi \alpha\|_{s+\frac{j}{2}, \mathcal{M}}
$$

holds and $\left(\tilde{\alpha}_{j}-\alpha\right) \wedge \bar{\partial} \rho=0$ on $\mathcal{M} \cap U_{0}$. By (3.7), (3.10) and the Sobolev interpolation theorem, we see that (3.1), (3.2) and (3.11) hold for $j=1$.

If we replace $\tilde{\alpha}_{j}$ by $\chi \tilde{\alpha}_{j}$, we may assume that $\tilde{\alpha}_{j} \in C_{0}^{\infty}\left(U_{0}\right)$ as before. Let us write:

$$
\begin{aligned}
\bar{\partial} \tilde{\alpha}_{j} & =\sum_{\substack{|I|=q-j+1,|J|=j \\
I \subset \mathcal{K}, J \subset \mathcal{K}^{c}}} \beta_{I J}^{j} d \bar{z}^{I} \wedge d \bar{z}^{J}+\sum_{\substack{|I|+|J|=q+1,|J| \geq j+1 \\
I \subset \mathcal{K}, J \subset \mathcal{K}^{c}}} E_{I J}^{j+1} d \bar{z}^{I} \wedge d \bar{z}^{J} \\
& :=\beta^{j}+E^{j+1} .
\end{aligned}
$$

For each fixed $J$ with $|J|=j \geq 1$, set

$$
\beta_{J}^{j}=\sum_{\substack{|I|=q-j+1 \\ I \subset \mathcal{K}}} \beta_{I J}^{j} d \bar{z}^{I}
$$

If we consider the terms in $\bar{\partial}^{2} \tilde{\alpha}_{j}$ with $|J|=j \geq 1, J \subset \mathcal{K}^{c}$, we see that

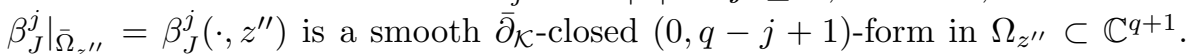
Since $\vartheta_{\mathcal{K}}=-*_{\mathcal{K}} \bar{\partial}_{\mathcal{K}} * \mathcal{K}$, it follows that $*_{\mathcal{K}} \beta_{J}^{j}$ is a $\vartheta_{\mathcal{K}}$-closed $(q+1, j)$-form in $\Omega_{z^{\prime \prime}} \subset \mathbb{C}^{q+1}$ for each $z^{\prime \prime} \in B_{\sigma_{0}}\left(z_{0}^{\prime \prime}\right)=I$.

Set

$$
u_{J}^{j}\left(\cdot, z^{\prime \prime}\right)=-*_{\mathcal{K}} \bar{\partial}_{\mathcal{K}} N_{(q+1, j)}^{\mathcal{K}} * \mathcal{K} \beta_{J}^{j}\left(\cdot, z^{\prime \prime}\right),
$$

where $N_{(q+1, j)}^{\mathcal{K}}$ is the Neumann operator for $(q+1, j)$-forms on the strongly pseudoconvex domains $\Omega_{z^{\prime \prime}} \subset \mathbb{C}^{q+1}$. Thus $u_{J}^{j}\left(\cdot, z^{\prime \prime}\right) \in \mathcal{C}^{0, q-j}\left(\Omega_{z^{\prime \prime}}\right)$, varying smoothly on $z^{\prime \prime} \in I$, and for each real $s \geq 0$ and nonnegative integer $k$, it follows from (2.1) and (3.11) that

$$
\left\|u_{J}^{j}\right\|_{s+\frac{1}{2}, k, U^{-}} \lesssim \sum_{r=0}^{k}\left\|\beta_{J}^{j}\right\|_{s+k-r, r, U^{-}} \lesssim\left\|\tilde{\alpha}_{j}\right\|_{s+k+1, U^{-}} \lesssim\|\chi \alpha\|_{s+k+\frac{j+1}{2}, \mathcal{M}} .
$$

Here, we need $s \geq 0$ (rather than $s \geq-1 / 2$ ) because $\beta_{J}^{j}$ may contain terms in $\bar{\partial}_{\mathcal{K}^{c}} \tilde{u}_{j-1}$ that can be estimated only in Sobolev $s$-norm in $\mathbb{C}^{q+1}$ for $s \geq 0$.

As for the $j=1$ case, we have to correct $u_{J}^{j}$ so that the corrected one, $\tilde{u}_{J}^{j}$, belongs to $\mathcal{C}^{0, q-j}\left(U_{0}\right)$, that is, $\tilde{u}_{J}^{j} \wedge \bar{\partial} \rho=0$ on $\mathcal{M} \cap U_{0}$. Since $u_{J}^{j} \wedge \bar{\partial}_{\mathcal{K}} \rho=0$ on $\mathcal{M} \cap U_{0}$, we can write

$$
u_{J}^{j}\left(\cdot, z^{\prime \prime}\right)=\delta_{J}^{j}\left(\cdot, z^{\prime \prime}\right) \wedge \bar{\partial}_{\mathcal{K}} \rho\left(\cdot, z^{\prime \prime}\right)+\rho\left(\cdot, z^{\prime \prime}\right) \gamma_{J}^{j}\left(\cdot, z^{\prime \prime}\right) \quad z^{\prime \prime} \in I
$$

for some $\delta_{J}^{j}\left(\cdot, z^{\prime \prime}\right) \in \mathcal{C}^{0, q-j-1}\left(\Omega_{z^{\prime \prime}}\right)$ and $\gamma_{J}^{j}\left(\cdot, z^{\prime \prime}\right) \in \mathcal{C}^{0, q-j}\left(\Omega_{z^{\prime \prime}}\right), z^{\prime \prime} \in I$. Set $\tilde{u}_{J}^{j}=u_{J}^{j}+\delta_{J}^{j} \wedge \bar{\partial}_{\mathcal{K}^{c}} \rho$. Then it follows that

$$
\tilde{u}_{J}^{j} \wedge \bar{\partial} \rho=\left(\delta_{J}^{j} \wedge \bar{\partial}_{\mathcal{K}} \rho+\rho \gamma_{J}^{j}+\delta_{J}^{j} \wedge \bar{\partial}_{\mathcal{K}^{c}} \rho\right) \wedge \bar{\partial} \rho=0
$$


on $\mathcal{M} \cap U_{0}$ for each $J \subset \mathcal{K}^{c}$ with $|J|=j$, and hence $\tilde{u}_{j}:=\sum_{J \subset \mathcal{K}^{c},|J|=j} \tilde{u}_{J}^{j} \wedge$ $d \bar{z}^{J} \in \mathcal{C}^{0, q}\left(U_{0} \cap \Omega\right)$. Also, one obtains that

$$
\begin{aligned}
\bar{\partial} \tilde{u}_{j} & =\sum_{J \subset \mathcal{K}^{c},|J|=j}\left(\beta_{J}^{j}+\bar{\partial}_{\mathcal{K}^{c}} \tilde{u}_{J}^{j}+\bar{\partial}\left(\delta_{J}^{j} \wedge \bar{\partial}_{\mathcal{K}^{c}} \rho\right)\right) \wedge d \bar{z}^{J} \\
& :=\sum_{J \subset \mathcal{K}^{c},|J|=j} \beta_{J}^{j} \wedge d \bar{z}^{J}+\tilde{E}^{j+1} .
\end{aligned}
$$

Set $\tilde{\alpha}_{j+1}=\tilde{\alpha}_{j}-\tilde{u}_{j} \in C^{\infty}\left(U_{0}\right)$. Note that we may assume that $\delta_{J}^{j} \wedge \bar{\partial}_{\mathcal{K}} \rho$ and $\rho \gamma_{J}^{j}$ are disjoint. Therefore, it follows, from the estimates in (3.11) and (3.12), and by the Sobolev interpolation theorem, that

$$
\left\|\tilde{\alpha}_{j+1}\right\|_{s+\frac{1}{2}, U^{-}} \lesssim\|\chi \alpha\|_{s+\frac{j+1}{2}, \mathcal{M}}
$$

for each real $s \geq 0$. Also, $\left(\tilde{\alpha}_{j+1}-\tilde{\alpha}_{j}\right) \wedge \bar{\partial} \rho=-\tilde{u}_{j} \wedge \bar{\partial} \rho=0$ on $\mathcal{M} \cap U_{0}$ since $\tilde{u}_{j} \in \mathcal{C}^{0, q}\left(U_{0} \cap \Omega\right)$. In view of $(3.13)$, we can write:

$$
\bar{\partial} \tilde{\alpha}_{j+1}=\sum_{\substack{|I|=q-j,|J|=j+1 \\ I \subset \mathcal{K}, J \subset \mathcal{K}^{c}}} \beta_{I J}^{j+1} d \bar{z}^{I} \wedge d \bar{z}^{J}+\sum_{\substack{|I|+|J|=q+1,|J| \geq j+2 \\ I \subset \mathcal{K}, J \subset \mathcal{K}^{c}}} E_{I J}^{j+2} d \bar{z}^{I} \wedge d \bar{z}^{J}
$$

for some smooth functions $\beta_{I J}^{j+1}$ and $E_{I J}^{j+2}$. This fact, together with the estimate in $(3.14)$, proves the inductive step. If we proceed up to $j=q+1$, then the proof of the proposition is completed.

Remark 3.2. When $n=q+1+k, 1 \leq k \leq q$, the above inductive step will stop at the $(k+1)$-th step, thus proving $\bar{\partial} \tilde{\alpha}_{k+1}=0$. This proves Theorem 1.1 with better estimates.

Now we are ready to prove the weak $\bar{\partial}$-closed extension problem (Theorem 1.1). We recall that $U_{0}^{-}=\bigcup_{z^{\prime \prime} \in I} \bar{\Omega}_{z^{\prime \prime}} \times\left\{z^{\prime \prime}\right\}$ as defined in (2.4).

Proof of Theorem 1.1. In view of Proposition 3.1, there exists $\alpha_{q+1} \in \bigwedge^{0, q}\left(U_{0}^{-}\right)$ which can be written as:

$$
\bar{\partial} \alpha_{q+1}=\sum_{\substack{J \subset \mathcal{K}^{c} \\|J|=q+1}} H_{J} d \bar{z}^{J}
$$

for some smooth functions $H_{J}$ on $U_{0}^{-}$. If we consider the coefficients of $d \bar{z}_{i} \wedge d \bar{z}^{J}$ of $\bar{\partial}^{2} \alpha_{q+1}=0$, we see that

$$
\frac{\partial H_{J}}{\partial \bar{z}_{i}}=0
$$

for $1 \leq i \leq q+1$. Hence $H_{J}\left(\cdot, z^{\prime \prime}\right)$ is a holomorphic function on $\Omega_{z^{\prime \prime}}$ for each $z^{\prime \prime} \in I$.

Also note that $\left(\alpha_{q+1}-\alpha\right) \wedge \bar{\partial} \rho=0$ on $\mathcal{M} \cap U_{0}$. Therefore, $\bar{\partial}\left(\alpha_{q+1}-\alpha\right) \wedge \bar{\partial} \rho=0$ and hence $\bar{\partial} \alpha_{q+1} \wedge \bar{\partial} \rho=0$ on $\mathcal{M} \cap U_{0}$ since $\bar{\partial}_{b} \alpha=0$ on $\mathcal{M} \cap U_{0}$. Considering 
the coefficients of $d \bar{z}_{i} \wedge d \bar{z}^{J}$ of $\bar{\partial} \alpha_{q+1} \wedge \bar{\partial} \rho$, one obtains, from (3.15), that

$$
\frac{\partial \rho}{\partial \bar{z}_{i}} H_{J}=0
$$

for $1 \leq i \leq q+1$ on $\mathcal{M} \cap U_{0}$. Since $\bar{\partial}_{\mathcal{K}} \rho \neq 0$ on $b \Omega_{z^{\prime \prime}}$, at least one of $\partial \rho / \partial \bar{z}_{i}$, for $1 \leq i \leq q+1$, is not equal to zero, and hence $H_{J}\left(\cdot, z^{\prime \prime}\right)=0$ on $b \Omega_{z^{\prime \prime}}$. Thus it follows that $H_{J}\left(\cdot, z^{\prime \prime}\right) \equiv 0$ on $\Omega_{z^{\prime \prime}}$ because $H_{J}\left(\cdot, z^{\prime \prime}\right)$ is a holomorphic function on $\Omega_{z^{\prime \prime}}$ for each $z^{\prime \prime} \in I$. In view of (3.15), we thus obtain that $\bar{\partial} \alpha_{q+1}=0$. If we set $\tilde{\alpha}=\alpha_{q+1}$, then $\tilde{\alpha}$ satisfies the estimates (1.1) from the estimates in (3.2). This proves Theorem 1.1.

Proof of Theorem 1.2. Let $U_{0}^{-}$be the neighborhood constructed in Theorem 1.1. Then there is a weak $\bar{\partial}$-closed extension $\tilde{\alpha}$ of $\alpha$ onto $U_{0}^{-}$satisfying the estimate (1.1). By the lemma in Section 4 of [2], we can construct a small pseudoconvex domain $B \subset \subset U_{0}^{-}$with the property that $W:=B \cap \mathcal{M}$ is a neighborhood of $z_{0} \in \mathcal{M}$.

For each real $s \geq 0$, set $\tilde{u}_{s}=\bar{\partial}^{*} N_{s}^{B} \tilde{\alpha}$, where $N_{s}^{B}$ denotes the weighted $\bar{\partial}$-Neumann operator in $B$ with weight $e^{-t_{s}|z|^{2}}$ for sufficiently large $t_{s}>0$ depending on $s$. Then we have $\bar{\partial} \tilde{u}_{s}=\tilde{\alpha}$ in $B$, and it follows that

$$
\left\|\tilde{u}_{s}\right\|_{s, B} \leq C_{s}\|\tilde{\alpha}\|_{s, B} .
$$

Set $u_{s}=\tau \tilde{u}_{s}$, where $\tau$ is the projection in $\bigwedge^{p, q-1}(\mathcal{M})$ onto $\mathcal{B}^{p, q-1}(\mathcal{M})$ defined by first restricting a $(p, q-1)$-form $\phi$ in $\mathbf{C}^{n}$ to $\mathcal{M}$, then projecting the restriction to $\mathcal{B}^{p, q-1}(\mathcal{M})$. Then $\bar{\partial}_{b} u_{s}=\alpha$ on $W$ and if we use the estimates (1.1) and (3.16), and the trace theorem in Sobolev spaces, then we obtain that

$$
\left\|u_{s}\right\|_{s, W} \leq C_{s}\left\|D \tilde{u}_{s}\right\|_{s-\frac{1}{2}, B} \leq C_{s}\|\tilde{\alpha}\|_{s+\frac{1}{2}, B} \leq C_{s}\|\chi \alpha\|_{s+\frac{q+1}{2}, U_{0}^{-} \cap \mathcal{M}}
$$

for each real $s \geq 0$. This completes the proof of Theorem 1.2.

\section{References}

[1] A. Andreotti and C. D. Hill, E. E. Levi convexity and the Hans Lewy problem. I, II, Ann. Scuola Norm. Sup. Pisa (3) 26 (1972), 325-363; ibid. 26 (1972), 747-806.

[2] S. Bell, Differentiability of the Bergman kernel and pseudo-local estimates, Math. Z. 192 (1986), 467-472.

[3] H. P. Boas and M.-C. Shaw, Sobolev estimates for the Lewy operator on weakly pseudoconvex boundaries, Math. Ann. 274 (1986), no. 2, 221-231.

[4] A. Boggess and M.-C. Shaw, A kernel approach to the local solvability of the tangential Cauchy Riemann equations, Trans. Amer. Math. Soc. 289 (1985), no. 2, 643-658.

[5] D. Catlin, Sufficient conditions for the extension of CR structures, J. Geom. Anal. 4 (1994), no. 4, 467-538.

[6] D. Catlin and S. Cho, Extension of CR structures on three dimensional compact pseudoconvex CR manifolds, Math. Ann. 334 (2006), no. 2, 253-280.

[7] S.-C. Chen and M.-C. Shaw, Partial Differential equations in several complex variables, AMS/IP Studies in Advanced Mathematics, 19. American Mathematical Society, Providence, RI; International Press, Boston, MA, 2001.

[8] S. Cho and J. Choi, Local extension of boundary holomorphic forms on real hypersurfaces in $\mathbb{C}^{n}$, J. Math. Anal. Appl. 325 (2007), no. 1, 279-293. 
[9] — Explicit Sobolev estimates for the Cauchy-Riemann equation on parameters, Bull. Korean Math. Soc. 45 (2008), no. 2, 321-338.

[10] S. Cho, Sobolev estimates for the local extension of $\bar{\partial}_{b}$-closed $(0,1)$-forms on real hypersurfaces in $\mathbb{C}^{n}$, Proc. of Amer. Math. Soc. 139 (2011), no. 11, 4053-4062.

[11] P. C. Greiner, J. J. Kohn, and E. M. Stein, Necessary and sufficient conditions for solvability of the Lewy equation, Proc. Nat. Acad. Sci. U.S.A. 72 (1975), no. 9, 32873289.

[12] R. S. Hamilton, Deformation of complex structures on manifolds with boundary I, II, J. Diff. Geom. 12 (1977), no. 1, 1-15; 14 (1979), no. 3, 409-473.

[13] G. M. Henkin and J. Leiterer, Andreotti-Grauert theory by Integral Formulas, Birkhäuser, Boston, Basel, 1988.

[14] J. J. Kohn and H. Rossi, On the extension of holomorphic functions from the boundary of a complex manifold, Ann. of Math. (2) 81 (1965), 451-472.

[15] M. Kuranishi, Strongly pseudoconvex CR structures over small balls. I. An a priori estimate, Ann. of Math. (2) 115 (1982), no. 3, 451-500.

[16] C. Laurent-Thiébaut and J. Leiterer, On the Hartogs-Bochner extension phenomenon for differential forms, Math. Ann. 284 (1989), no. 1, 103-119.

[17] H. Lewy, On the local character of the solution of an atypical linear differential equation in three variables and a related theorem for regular functions of two complex variables, Ann. of Math. (2) 64 (1956), 514-522.

[18] An example of a smooth linear partial differential equation without solution, Ann. of Math. (2) 66 (1957), 155-158.

[19] M.-C. Shaw, $L^{2}$ estimates and existence theorems for the tangential Caucy-Riemann complex, Invent. Math. 82 (1985), no. 1, 133-150.

$[20] ـ, L^{2}$ existence theorems for the $\bar{\partial}_{b}-$ Neumann problem on strongly pseudoconvex CR manifolds, J. Geom. Anal. (2) 1 (1992), 139-163.

[21] S. Webster, On the proof of Kuranishi's embedding theorem, Ann. Inst. H. Poincaré Anal. Non Linéaire 6 (1989), no. 3, 183-207.

Department of Mathematics

SOGANG UNIVERSITY

SEOUL 121-742, KoreA

E-mail address: shcho@sogang.ac.kr 